\title{
Space-Time MIMO Channel Modelling using Angular Power Distributions
}

\author{
Terence Betlehem, Thushara D. Abhayapala ${ }^{\dagger}$ and Tharaka A. Lamahewa \\ Department of Telecommunications Engineering \\ Research School of Information Sciences and Engineering \\ The Australian National University \\ Canberra ACT 0200, Australia \\ \{terence.betlehem, thushara.abhayapala, tharaka.lamahewa\}eanu.edu.au
}

\begin{abstract}
In this paper, we develop a MIMO channel model for generating the channel gains between arbitrary arrays of transmitter and receiver antennas, for a general class of non-lineof-sight (NLOS) channels. The channel scattering environment is defined by a double directional angular distribution describing the power transferred from transmitter aperture to receiver aperture in each direction. We propose several parametrized bivariate distributions that are consistent with univariate scatterer distributions separately observed at the transmitter and receiver. We derive the second order statistics of the channel gains in terms of the double directional power distribution and characterize a sample system performance as a function of distribution parameters.
\end{abstract}

\section{INTRODUCTION}

With recent development of practical MIMO systems, there is need to quantify MIMO system performance over realistic channels. Many options for channel models are now available but either require ray-tracing, complicated parametrizations, or restrict simulation to fixed array geometries at transmitter and receiver. We present a model for arbitrary array geometries that can be used to generate channel realizations from double directional power distribution data.

A number of schemes have been proposed for modelling MIMO channels. Several authors propose ray tracing models [1]. For non-line-of-sight channels, the channel gains are dominated by their second order statistics [2]. To model the second order channel statistics, many use oversimplified models such as Rayleigh fading and Kronecker models [3], [4] which poorly estimate capacity [5]. Others use higher complexity data-dependent models (e.g. [6], [7]) which learn statistical parameters from a particular data set, or geometric models [8] based on parametrizations of the directional power distribution.

In this paper, we describe a model for non-line-of-sight channels based upon the directional power distribution of scatterers. As little work has been put towards exploring plausible bi-directional distributions, we explore some distributions such as the elliptical bivariate equivalents to the Gaussian and Laplacian distributions, quantifying MIMO system capacity and diversity as a function of bivariate parameters. The advantages of our model are that (i) arbitrary geometries of

This work was partially funded by Australian Research Council Discovery Grant number DP0343804.

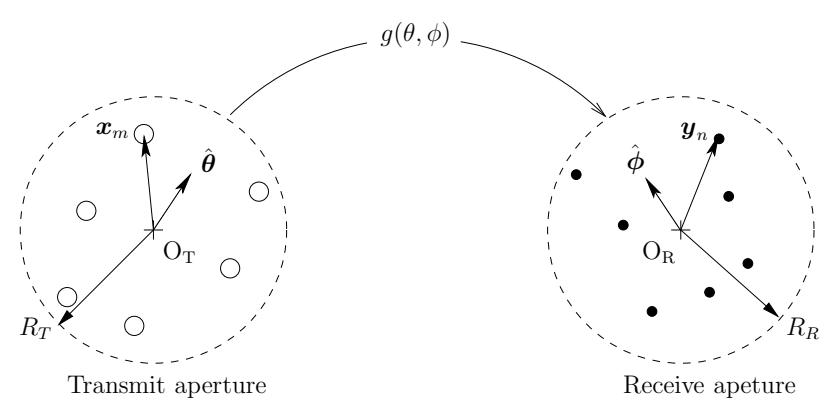

1: The channel model. Scattering is modelled with the scattering gain $g(\theta, \phi)$. Each transmitter (o) is positioned at $\boldsymbol{x}_{m}$ within a circular aperture of radii $R_{T}$ and each receiver $(\cdot)$ at $\boldsymbol{y}_{n}$ within a circular aperture of radius $R_{R}$.

transmitters and receivers can be simulated from the same set of data, (ii) it is derived from an efficient parametrization of the channel guaranteeing a mimimal modelling order. We present a 2-D model, which extends naturally to 3-D.

\section{Channel Model}

Consider transmission of data from $n_{T}$ transmitter antennas over a flat fading channel to $n_{R}$ receiver antennas. Let $\boldsymbol{x}_{m}$ be the $m$ th transmitter antenna position with respect to an transmitter origin $\mathrm{O}_{\mathrm{T}}$ and $\boldsymbol{y}_{n}$ be the $n$th receiver antenna position with respect to a receiver origin $\mathrm{O}_{R}$ (as shown in Fig. 1). Transmitter and receiver antennas lie within finite circular apertures of radius $R_{T}$ and $R_{R}$ respectively. Each transmitter $m$ transmits a signal $s_{m}(t)$ over the time-varying channel with the transfer function $h_{n m}(t)$ to generate a signal $z_{n}(t)$ at each receiver $n$. Collect transfer functions into a matrix $\boldsymbol{H}(t)$ such that $[\boldsymbol{H}]_{n m} \triangleq h_{n m}$, transmitted signals into $\boldsymbol{s}(t) \triangleq\left[s_{1}(t), s_{2}(t), \ldots s_{n_{T}}(t)\right]^{T}$ where $\cdot^{T}$ is the vector transpose operator, and received signals $z_{n}(t)$ into vector $\boldsymbol{z}(t) \triangleq\left[z_{1}(t), z_{2}(t), \ldots z_{n_{R}}(t)\right]^{T}$. Letting $\boldsymbol{w}(t) \triangleq$ $\left[w_{1}(t), w_{2}(t), \ldots w_{n_{R}}(t)\right]^{T}$ be additive white Gaussian noise at the receivers, we write:

$$
\boldsymbol{z}(t)=\boldsymbol{H}(t) \boldsymbol{s}(t)+\boldsymbol{w}(t) .
$$

Assume that all scatterers lie in the far-field. The scattering environment causes transmitter signals to propagate in as plane waves with a different amplitude for each direction. Define the scattering gain $g(\theta, \phi, t)$ as the complex gain at time $t$ of the signal propagating out from the transmitter origin in direction 
$\theta$ and arriving at the receiver origin in direction $\phi$. We can then write:

$$
h_{n m}(t)=\int_{2 \pi} \int_{2 \pi} g(\theta, \phi, t) e^{i k\left(\boldsymbol{x}_{m} \cdot \hat{\boldsymbol{\theta}}-\boldsymbol{y}_{n} \cdot \hat{\boldsymbol{\phi}}\right)} d \phi
$$

where $\hat{\boldsymbol{\theta}}$ and $\hat{\boldsymbol{\phi}}$ are unit vectors of polar coordinates $(1, \phi)$ and $(1, \theta)$ and $k$ is the wave number. Assume slow fading so that the channel remains static over the symbol time. Then $h_{n m}$ and $g$ are not dependent on $t$ over each symbol.

To investigate the statistics of non-line-of-sight scattering, $g(\theta, \phi)$ is assumed to be a zero mean Gaussian random variable at each angle pair $(\theta, \phi)$. Further assume $g(\theta, \phi)$ uncorrelated between different angles:

$$
E\left\{g(\theta, \phi) g^{*}(\vartheta, \varphi)\right\}=\mathcal{P}(\theta, \phi) \delta_{\theta \vartheta} \delta_{\phi \varphi},
$$

where $\delta_{\phi \varphi}$ is the Kronecker delta function and $*^{*}$ is the complex conjugate and $\mathcal{P}(\theta, \phi) \triangleq E\left\{|g(\theta, \phi)|^{2}\right\}$ is the power density of scatterers, interpreted as the average energy transmitted from transmitter aperture in direction $\theta$ arriving at receiver aperture in direction $\phi$. This assumption is referred to as the wide-sense stationary uncorrelated scatterer (WSSUS) assumption [9]. Without loss of generality, we assume $\mathcal{P}(\theta, \phi)$ is normalized:

$$
\int_{2 \pi} \int_{2 \pi} \mathcal{P}(\theta, \phi) d \theta d \phi=1 .
$$

Statistics of $g(\theta, \phi)$ and hence $\boldsymbol{H}$ are dependent entirely upon $\mathcal{P}(\theta, \phi)$.

The univariate marginals of the angular power are understood as the power density of scatterers at the transmitter:

$$
\mathcal{P}_{T}(\theta) \triangleq \int_{2 \pi} \mathcal{P}(\theta, \phi) d \phi,
$$

and power density of scatterers at the receiver:

$$
\mathcal{P}_{R}(\phi) \triangleq \int_{2 \pi} \mathcal{P}(\theta, \phi) d \theta .
$$

Suitable functional forms for these scatterer distributions have been well-studied [10]-[13]. These distributions characterize the properties of the separable Kronecker model [4].

\section{Channel PARAMETRIZATION}

We now derive a simple structure in the parameters of the above channel model. By transforming angular functions into the Fourier domain, we show how to represent the channel mixing matrix $\boldsymbol{H}$ with a minimal number of parameters. We then derive the statistics of the Fourier parameters.

\section{A. Fourier Expansion}

Perform the double Fourier expansion of $\mathcal{P}(\theta, \phi)$,

$$
\begin{gathered}
\mathcal{P}(\theta, \phi)=\frac{1}{(2 \pi)^{2}} \sum_{\ell=-\infty}^{\infty} \sum_{\ell^{\prime}=-\infty}^{\infty} \gamma_{\ell \ell^{\prime}} e^{i\left(\ell \theta-\ell^{\prime} \phi\right)}, \\
\gamma_{\ell \ell^{\prime}}=\int_{2 \pi} \int_{2 \pi} \mathcal{P}(\theta, \phi) e^{-i\left(\ell \theta-\ell^{\prime} \phi\right)} d \theta d \phi .
\end{gathered}
$$

where $\gamma_{\ell \ell^{\prime}}$ is the Fourier coefficient of $\mathcal{P}(\theta, \phi)$. Since $\mathcal{P}(\theta, \phi) \in \mathbb{R}$, we know that $\gamma_{\ell\left(-\ell^{\prime}\right)}^{*}=\gamma_{(-\ell) \ell^{\prime}}$. Also note that $\mathcal{P}(\theta, \phi) \geq 0$ which is conservatively satisfied by the condition:

$$
\gamma_{00} \geq 2 \sum_{\ell=1}^{\infty} \sum_{\ell^{\prime}=1}^{\infty}\left|\gamma_{\ell \ell^{\prime}}\right| .
$$

Substituting (5a) into (4) we see the power density of scatterers around transmitter and receiver are just a function of the $\gamma_{\ell 0}$ and $\gamma_{0 \ell^{\prime}}$ coefficients:

$$
\begin{gathered}
\mathcal{P}_{T}(\theta)=\frac{1}{2 \pi} \sum_{\ell=-\infty}^{\infty} \gamma_{\ell 0} e^{i \ell \theta}, \\
\mathcal{P}_{R}(\phi)=\frac{1}{2 \pi} \sum_{\ell^{\prime}=-\infty}^{\infty} \gamma_{0 \ell^{\prime}} e^{-i \ell^{\prime} \theta} .
\end{gathered}
$$

Similarly write $g(\theta, \phi)$ as the double Fourier expansion:

$$
\begin{gathered}
g(\theta, \phi)=\frac{1}{(2 \pi)^{2}} \sum_{\ell=-\infty}^{\infty} \sum_{\ell^{\prime}=-\infty}^{\infty} \beta_{\ell \ell^{\prime}} e^{i\left(\ell \theta-\ell^{\prime} \phi\right)}, \\
\beta_{\ell \ell^{\prime}}=\int_{2 \pi} \int_{2 \pi} g(\theta, \phi) e^{-i\left(\ell \theta-\ell^{\prime} \phi\right)} d \theta d \phi,
\end{gathered}
$$

where $\beta_{\ell \ell^{\prime}}$ is the Fourier coefficient of $g(\theta, \phi)$. Due to the limited aperture sizes, (7a) can be truncated and from (2) each $h_{n m}(t)$ written as a sum of a small number of $\beta_{\ell \ell^{\prime}}$ coefficients. Drawing from [14], we state the theorem:

Theorem 1 (General MIMO Parametrization): For sets of $n_{T}$ transmitter and $n_{R}$ receiver antennas, where each is positioned at $\boldsymbol{x}_{m}$ and $\boldsymbol{y}_{n}$ within circular apertures of radii $R_{T}$ and $R_{R}$ respectively, the matrix of channel weights $\boldsymbol{H}$ defined in MIMO model (1) can be decomposed:

$$
\boldsymbol{H}=\boldsymbol{J}_{\boldsymbol{R}} \boldsymbol{\beta} \boldsymbol{J}_{\boldsymbol{T}}^{\dagger},
$$

where ${ }^{\dagger}$ is the hermitian operator,

$$
\boldsymbol{\beta}=\left[\begin{array}{ccc}
\beta_{\left(-N_{R}\right)\left(-N_{T}\right)} & \cdots & \beta_{\left(-N_{R}\right) N_{T}} \\
\beta_{\left(-N_{R}+1\right)\left(-N_{T}\right)} & \cdots & \beta_{\left(-N_{R}+1\right) N_{T}} \\
\vdots & \ddots & \vdots \\
\beta_{N_{R}\left(-N_{T}\right)} & \cdots & \beta_{N_{R} N_{T}}
\end{array}\right],
$$

is the matrix of the scattering function coefficients defined in (7a), $\boldsymbol{J}_{\boldsymbol{T}}$ and $\boldsymbol{J}_{\boldsymbol{R}}$ are the transmitter and receiver antenna sampling matrices

$$
\boldsymbol{J}_{\boldsymbol{T}}=\left[\begin{array}{ccc}
\mathcal{J}_{-N_{T}}\left(\boldsymbol{x}_{1}\right) & \ldots & \mathcal{J}_{N_{T}}\left(\boldsymbol{x}_{1}\right) \\
\vdots & \ddots & \vdots \\
\mathcal{J}_{-N_{T}}\left(\boldsymbol{x}_{n_{T}}\right) & \cdots & \mathcal{J}_{N_{T}}\left(\boldsymbol{x}_{n_{T}}\right),
\end{array}\right]
$$

and

$$
\boldsymbol{J}_{\boldsymbol{R}}=\left[\begin{array}{ccc}
\mathcal{J}_{-N_{R}}\left(\boldsymbol{y}_{1}\right) & \ldots & \mathcal{J}_{N_{R}}\left(\boldsymbol{y}_{1}\right) \\
\vdots & \ddots & \vdots \\
\mathcal{J}_{-N_{R}}\left(\boldsymbol{y}_{n_{R}}\right) & \ldots & \mathcal{J}_{N_{R}}\left(\boldsymbol{y}_{n_{R}}\right)
\end{array}\right],
$$

and if vector $\boldsymbol{x}$ is defined in polar coordinates as $\left(x, \theta_{x}\right)$, then

$$
\mathcal{J}_{\ell}(\boldsymbol{x}) \triangleq(-i)^{\ell} J_{\ell}(k x) e^{i \ell \theta_{x}},
$$

$N_{T}=\left\lceil e k R_{T} / 2\right\rceil$ and $N_{R}=\left\lceil e k R_{R} / 2\right\rceil$ are the aperture dimensionalities of transmiter and receiver respectively and $J_{m}(\cdot)$ is the Bessel function of the first kind of order $m$.

Proof for this theorem is in [14] and is not repeated here. 


\section{B. Statistical Relationships}

To model the channel matrix $\boldsymbol{H}$ we characterize the statistics of $\boldsymbol{\beta}$. Since the scattering gain is zero mean Gaussian, the statistics of the elements of $\boldsymbol{\beta}$ are governed by its correlation matrix $\boldsymbol{R}_{\boldsymbol{\beta}} \triangleq E\left\{\overrightarrow{\boldsymbol{\beta}} \overrightarrow{\boldsymbol{\beta}}^{\dagger}\right\}$ where vectorize operator $\vec{r}$ stacks the columns of a matrix. From (7b) each second order statistic $E\left\{\beta_{p p^{\prime}} \beta_{q q^{\prime}}^{*}\right\}$ can be calculated as:

$$
\begin{aligned}
E\left\{\beta_{p p^{\prime}} \beta_{q q^{\prime}}^{*}\right\}= & \int_{2 \pi} \int_{2 \pi} \int_{2 \pi} \int_{2 \pi} e^{-i\left(p \theta-p^{\prime} \phi\right)} e^{i\left(q \vartheta-q^{\prime} \varphi\right)} \\
& \times E\left\{g(\theta, \phi) g^{*}(\vartheta, \varphi)\right\} d \theta d \phi d \vartheta d \varphi
\end{aligned}
$$

Applying the WSSUS property (3):

$$
E\left\{\beta_{p p^{\prime}} \beta_{q q^{\prime}}^{*}\right\}=\int_{2 \pi} \int_{2 \pi} \mathcal{P}(\theta, \phi) e^{-i(p-q) \theta} e^{i\left(p^{\prime}-q^{\prime}\right) \phi} d \theta d \phi .
$$

We see by comparison of (13) with (5b) that:

$$
E\left\{\beta_{p p^{\prime}} \beta_{q q^{\prime}}^{*}\right\}=\gamma_{(p-q)\left(p^{\prime}-q^{\prime}\right)},
$$

from which is written:

Theorem 2 (WSSUS Channel Correlation): In a WSSUS channel possessing angular power distribution $\mathcal{P}(\theta, \phi)$, the correlation matrix $\boldsymbol{R}_{\boldsymbol{\beta}} \triangleq E\left\{\overrightarrow{\boldsymbol{\beta}} \overrightarrow{\boldsymbol{\beta}}^{\dagger}\right\}$ of $\boldsymbol{\beta}$ defined in (7b) possesses the block Toeplitz structure:

$$
\boldsymbol{R}_{\boldsymbol{\beta}}=\left[\begin{array}{cccc}
\boldsymbol{\Gamma}_{0} & \boldsymbol{\Gamma}_{-1} & \ldots & \boldsymbol{\Gamma}_{-2 N_{T}} \\
\boldsymbol{\Gamma}_{1} & \boldsymbol{\Gamma}_{0} & \ldots & \boldsymbol{\Gamma}_{-2 N_{T}+1} \\
\vdots & \vdots & \ddots & \vdots \\
\boldsymbol{\Gamma}_{2 N_{T}} & \boldsymbol{\Gamma}_{2 N_{T}-1} & \ldots & \boldsymbol{\Gamma}_{0}
\end{array}\right]
$$

and $\boldsymbol{\Gamma}_{\ell}$ is Toeplitz:

$$
\boldsymbol{\Gamma}_{\ell}=\left[\begin{array}{cccc}
\gamma_{0 \ell} & \gamma_{(-1) \ell} & \ldots & \gamma_{\left(-2 N_{R}\right) \ell} \\
\gamma_{1 \ell} & \gamma_{0 \ell} & \ldots & \gamma_{\left(-2 N_{R}+1\right) \ell} \\
\vdots & \vdots & \ddots & \vdots \\
\gamma_{2 N_{R} \ell} & \gamma_{\left(2 N_{R}-1\right) \ell} & \ldots & \gamma_{0 \ell}
\end{array}\right]
$$

and $\gamma_{\ell \ell^{\prime}}$ is defined in $(5 \mathrm{~b})$.

Comments:

- The parametrizations in the above theorems allow abstraction of the channel outside the apertures from the array geometries inside.

- The statistics of $\boldsymbol{H}$ are completely governed by covariance $\boldsymbol{R}_{\boldsymbol{\beta}}$ of $\boldsymbol{\beta}$. From (8) and Kronecker relation [15] $\overrightarrow{\boldsymbol{A B C}}=\left(\boldsymbol{A}^{T} \otimes \boldsymbol{C}\right) \overrightarrow{\boldsymbol{B}}$ where $\otimes$ is the Kronecker product operator, the channel correlation matrix $\boldsymbol{R}_{\boldsymbol{H}} \triangleq$ $E\left\{\overrightarrow{\boldsymbol{H}} \overrightarrow{\boldsymbol{H}}^{\dagger}\right\}$ is calculated from $\boldsymbol{R}_{\boldsymbol{\beta}}$ through

$$
\boldsymbol{R}_{\boldsymbol{H}}=\left(\boldsymbol{J}_{\boldsymbol{T}}^{*} \otimes \boldsymbol{J}_{\boldsymbol{R}}\right) \boldsymbol{R}_{\boldsymbol{\beta}}\left(\boldsymbol{J}_{\boldsymbol{T}}^{T} \otimes \boldsymbol{J}_{\boldsymbol{R}}^{\dagger}\right) .
$$

- Although $\boldsymbol{R}_{\boldsymbol{\beta}}$ is of a large dimension $\left(2 N_{T}+1\right)\left(2 N_{R}+\right.$ 1) $\times\left(2 N_{T}+1\right)\left(2 N_{R}+1\right)$, we are able to completely describe the channel statistics for any antenna geometries within the transmitter and receiver apertures with only $\left(2 N_{T}+1\right)\left(2 N_{R}+1\right)$ complex parameters.

- By virtue of the $\gamma_{\ell\left(-\ell^{\prime}\right)}=\gamma_{(-\ell) \ell^{\prime}}^{*}$ property, $\boldsymbol{\Gamma}_{-\ell}=\boldsymbol{\Gamma}_{\ell}^{\dagger}$.

\section{MiMO System SimulatoR}

The above theorems motivate the following procedure for simulating a MIMO system defined by parameters $\left\{\boldsymbol{x}_{m}\right\}$, $\left\{\boldsymbol{y}_{n}\right\}$ and $\left\{\gamma_{\ell \ell^{\prime}}\right\}$ :

1) Calculate the coefficient correlation matrix $\boldsymbol{R}_{\boldsymbol{\beta}}$ from (14), sampling matrices $\boldsymbol{J}_{\boldsymbol{T}}$ and $\boldsymbol{J}_{\boldsymbol{R}}$ from (10) and (11), and the channel correlation matrix $\boldsymbol{R}_{\boldsymbol{H}}$ through (15).

2) Calculate realizations of the channel matrix using

$$
\boldsymbol{H}=\boldsymbol{R}_{\boldsymbol{H}}^{1 / 2} \boldsymbol{H}_{\mathrm{iid}},
$$

where $\boldsymbol{H}_{\text {iid }}$ is an $n_{R} \times n_{T}$ matrix of i.i.d. zero mean unit variance circularly-symmetric complex Gaussian elements and $\boldsymbol{R}_{\boldsymbol{H}}{ }^{1 / 2}$ is obtained from Cholesky decomposition $\boldsymbol{R}_{\boldsymbol{H}}{ }^{1 / 2}\left[\boldsymbol{R}_{\boldsymbol{H}}{ }^{1 / 2}\right]^{\dagger}=\boldsymbol{R}_{\boldsymbol{H}}$.

This channel simulator is utilized in Section VI to characterize performance of a $10 \times 10$ MIMO system.

\section{Bi-Directional Power Distributions}

Although various power distributions have been proposed for the angular power around the receiver [10]-[13], and by the reciprocity principle of the wave equation the same distributions hold for the angular power around the transmitter, little has been suggested for the bi-directional distribution $\mathcal{P}(\theta, \phi)$.

We propose natural extension of the univariate distributions to the bi-directional case. The bi-directional distributions are parametrized by a mean angle-of-arrival / angle-of-departure direction pair $\boldsymbol{\theta}_{0} \triangleq\left(\theta_{0}, \phi_{0}\right)$, angular spread at the transmitter $\sigma_{t}$ and receiver $\sigma_{r}$, and a parameter controlling joint properties.

For a bivariate distribution, we choose a power distribution (i) whose marginal density functions are well-known and well-studied i.e., the uniform limited [11], Gaussian [12] and Laplacian [13] marginals, and (ii) has closed-form expressions for their Fourier coefficients.

We can calculate the Fourier coefficients of $\mathcal{P}(\theta, \phi)$ from the characteristic function $\chi_{f}\left(\ell, \ell^{\prime}\right)=E_{f}\left\{e^{i\left(\ell \theta+\ell^{\prime} \phi\right)}\right\}$ of the density function $f(\theta, \phi)=\mathcal{P}(\theta, \phi)$ :

$$
\chi_{f}\left(\ell, \ell^{\prime}\right)=\int_{-\infty}^{\infty} \int_{-\infty}^{\infty} e^{i\left(\ell \theta+\ell^{\prime} \phi\right)} f(\theta, \phi) d \theta d \phi
$$

If $f(\theta, \phi)$ is narrow- that is, if negligible energy lies outside of $\{(\theta, \phi):-\pi \leq \theta<\pi,-\pi \leq \phi<\pi\}-$ the integral can be truncated to $2 \pi$ and by comparison with $(5 b), \gamma_{\ell \ell^{\prime}}=$ $\chi_{f}\left(\ell,-\ell^{\prime}\right)$.

Below three families of bi-directional distributions are introduced. These are illustrated in Fig. 2.

\section{A. Morgenstern Distributed Scatterers}

In the case the energy leaves the transmitter aperture uniformly from $\left(\theta_{0}-\Delta_{t}, \theta_{0}+\Delta_{t}\right)$ to arrive at the receiver aperture uniformly from $\left(\theta_{0}-\Delta_{r}, \theta_{0}+\Delta_{r}\right)$, the marginal constraints (4a) and (4b) can be satisfied with Morgenstern's family of distributions [16]:

$$
f_{\text {Morg }}(\theta, \phi)=\frac{1}{4 \Delta_{t} \Delta_{r}}-\frac{\rho_{m}\left(\theta-\theta_{0}\right)\left(\phi-\phi_{0}\right)}{4 \Delta_{t}^{2} \Delta_{r}^{2}} .
$$




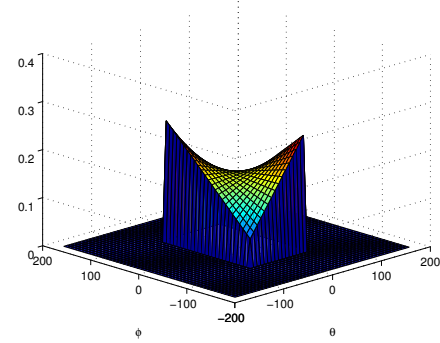

(a)

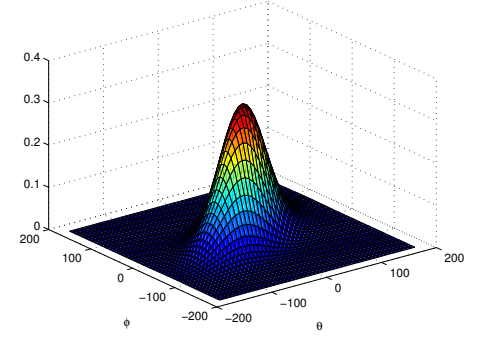

(b)

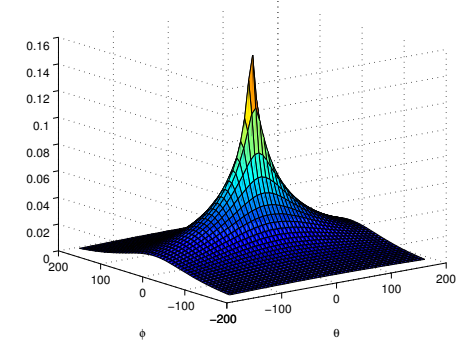

(c)

2: Shapes of proposed scatterer distributions: (a) Morgenstern $\left(\rho_{m}=0.7\right)$, (b) bivariate Gaussian $\left(\theta_{v}=\pi / 6, \sigma_{1}=2, \sigma_{2}=1\right)$ and (c) bivariate Laplacian $\left(\theta_{v}=\pi / 6, \sigma_{1}=4, \sigma_{2}=2\right)$.

Using (5b) it is straight-forward to derive without approximation:

$$
\gamma_{\ell \ell^{\prime}}=\left\{\begin{array}{cc}
e^{i \ell \theta_{0}} \operatorname{sinc}\left(\ell \Delta_{t}\right), & \ell^{\prime}=0, \\
e^{-i \ell^{\prime} \phi_{0}} \operatorname{sinc}\left(\ell^{\prime} \Delta_{r}\right), & \ell=0, \\
e^{i\left(\ell \theta_{0}-\ell^{\prime} \theta_{0}\right)} \Gamma\left(\ell, \ell^{\prime}\right), & \text { otherwise, }
\end{array}\right.
$$

where

$$
\begin{aligned}
\Gamma\left(\ell, \ell^{\prime}\right)= & \operatorname{sinc}\left(\ell \Delta_{t}\right) \operatorname{sinc}\left(\ell^{\prime} \Delta_{r}\right)-\frac{\rho_{m}}{\ell \Delta_{t} \ell^{\prime} \Delta_{r}} \times \\
& {\left[\cos \left(\ell \Delta_{t}\right)-\operatorname{sinc}\left(\ell \Delta_{t}\right)\right]\left[\cos \left(\ell^{\prime} \Delta_{r}\right)-\operatorname{sinc}\left(\ell^{\prime} \Delta_{r}\right)\right] . }
\end{aligned}
$$

Parameter $\rho_{m} \in[-1,1]$ controls the shape of $f_{\mathrm{Morg}}(\theta, \phi)$. In case $\rho_{m}=0$, the distribution reduces to the separable product of two uniform limited distributions. Further in the isotropic case $\Delta_{t}=\pi$ and $\Delta_{r}=\pi$ the Morgenstern distribution reduces to the rich scattering distribution $f_{\text {Iso }}(\theta, \phi)=1 /(2 \pi)^{2}$.

\section{B. Gaussian Distributed Scatterers}

The truncated bivariate Gaussian distribution is written: $f_{\mathrm{G}}(\theta, \phi)=K_{\mathrm{G}} e^{-\left(\boldsymbol{\theta}-\boldsymbol{\theta}_{0}\right)^{T} \boldsymbol{\Sigma}^{-1}\left(\boldsymbol{\theta}-\boldsymbol{\theta}_{0}\right) / 2},\left|\theta-\theta_{0}\right|,\left|\phi-\phi_{0}\right| \leq \pi$ where $\boldsymbol{\theta}=[\theta, \phi]^{T}$ and $K_{\mathrm{G}}$ is a normalization constant. It is an elliptically contoured function centered about $\boldsymbol{\theta}_{0}=\left[\theta_{0}, \phi_{0}\right]^{T}$ and parameterized in terms the $2 \times 2$ symmetric matrix $\boldsymbol{\Sigma}$ whose eigenvectors $\boldsymbol{v}_{1}$ and $\boldsymbol{v}_{2}$ are the directions of the major and minor axes of the distribution contours and eigenvalues $\sigma_{1}^{2}$ and $\sigma_{2}^{2}$ are the variances along major and minor axes:

$$
\boldsymbol{\Sigma} \triangleq\left[\begin{array}{cc}
\sigma_{t}^{2} & \rho_{e} \\
\rho_{e} & \sigma_{r}^{2}
\end{array}\right]=\left[\begin{array}{ll}
\boldsymbol{v}_{1} & \boldsymbol{v}_{2}
\end{array}\right]\left[\begin{array}{cc}
\sigma_{1}^{2} & 0 \\
0 & \sigma_{2}^{2}
\end{array}\right]\left[\begin{array}{c}
\boldsymbol{v}_{1}^{T} \\
\boldsymbol{v}_{2}^{T}
\end{array}\right]
$$

In this form, one can show that:

$$
\begin{aligned}
\sigma_{t}^{2} & =\sigma_{1}^{2}+\left(\sigma_{2}^{2}-\sigma_{1}^{2}\right) \sin ^{2} \theta_{v}, \\
\sigma_{r}^{2} & =\sigma_{2}^{2}+\left(\sigma_{1}^{2}-\sigma_{2}^{2}\right) \sin ^{2} \theta_{v}, \\
\rho_{e} & =\left(\sigma_{1}^{2}-\sigma_{2}^{2}\right) \sin \theta_{v} \cos \theta_{v},
\end{aligned}
$$

and $\theta_{v}$ is the angle between $\boldsymbol{v}_{1}$ and the $\theta$-axis controlling the orientation of the ellipse and here $\left|\rho_{e}\right| \leq\left(\sigma_{1}^{2}-\sigma_{2}^{2}\right) / 2$. The parameters are illustrated in Fig. 3. For small angular spread $\left(\sigma_{r}, \sigma_{t} \ll \pi\right), K_{\mathrm{G}}=1 / 2 \pi \Sigma^{1 / 2}$ and coefficients $\gamma_{\ell \ell^{\prime}}$ are obtained from the Gaussian characteristic function:

$$
\chi_{\mathrm{G}}\left(\ell,-\ell^{\prime}\right)=e^{i\left(\ell \theta_{0}-\ell^{\prime} \phi_{0}\right)} e^{-\frac{1}{2}\left[\ell^{2} \sigma_{t}^{2}+\ell^{2} \sigma_{r}^{2}-2 \rho_{e} \ell \ell^{\prime}\right]} .
$$

The marginal distributions possess characteristic functions of form $\chi_{\mathrm{G}}(\ell)=e^{i \ell \theta_{0}} e^{-\frac{1}{2} \ell^{2} \sigma_{t}^{2}}$, which correspond to the

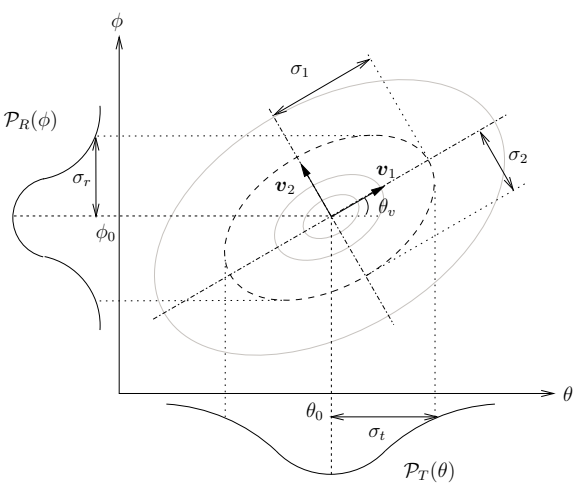

3: Contour plot for a typical elliptical bivariate scatterer power distribution, showing distribution parameters: mean direction pair $\left(\theta_{0}, \phi_{0}\right)$, major and minor axes of ellipses $\boldsymbol{v}_{1}$ and $\boldsymbol{v}_{2}$ and axes spread parameters $\sigma_{1}$ and $\sigma_{2}$, respectively. Also shown are the resulting scatterer densities $P_{T}(\theta)$ and $P_{R}(\phi)$.

univariate Gaussian distribution $f_{\mathrm{G}}(\theta)=\frac{1}{\sqrt{2 \pi} \sigma_{t}} e^{-\left(\theta-\theta_{0}\right)^{2} / 2 \sigma_{t}^{2}}$ [17].

For $\mathcal{P}(\theta, \phi)=f_{\mathrm{G}}(\theta, \phi)$, the variances in the marginals $\sigma_{t}^{2}$ and $\sigma_{r}^{2}$ are the variances in the angular spreads of $P_{T}(\theta)$ and $P_{R}(\phi)$ respectively.

\section{Laplacian Distributed Scatterers}

Similarly to the Gaussian distribution, a truncated elliptical bivariate Laplacian distribution can also be written [18]:

$$
\begin{array}{r}
f_{\mathrm{L}}(\theta, \phi)=K_{\mathrm{L}} \mathcal{K}_{0}\left(\sqrt{2\left(\boldsymbol{\theta}-\boldsymbol{\theta}_{0}\right)^{T} \boldsymbol{\Sigma}^{-1}\left(\boldsymbol{\theta}-\boldsymbol{\theta}_{0}\right)}\right) \\
\left|\theta-\theta_{0}\right|,\left|\phi-\phi_{0}\right| \leq \pi,
\end{array}
$$

where $\mathcal{K}_{0}(\cdot)$ is the modified Bessel function of the second kind of order zero and $K_{\mathrm{L}}$ is a normalization constant. For small angular spread, $K_{\mathrm{L}}=1 / \pi|\boldsymbol{\Sigma}|^{1 / 2}$ and coefficients are obtained from the Laplacian characteristic function [18]:

$$
\chi_{\mathrm{L}}\left(\ell,-\ell^{\prime}\right)=\frac{e^{i\left(\ell \theta_{0}-\ell^{\prime} \phi_{0}\right)}}{1+\sigma_{t}^{2} \ell^{2}+\sigma_{r}^{2} \ell^{2}-2 \rho_{e} \ell \ell^{\prime}} .
$$

The marginal distributions possess characteristic functions of form $\chi_{\mathrm{L}}(\ell)=e^{i \ell \theta_{0}} /\left(1+\sigma_{t}^{2} \ell^{2}\right)$ which correspond to the well-known univariate Laplacian distribution $f_{\mathrm{L}}(\theta)=$ $\frac{1}{2 \sigma_{t}} e^{-\left|\theta-\theta_{0}\right| / \sigma_{t}}$ with variance $2 \sigma_{t}^{2}$. 


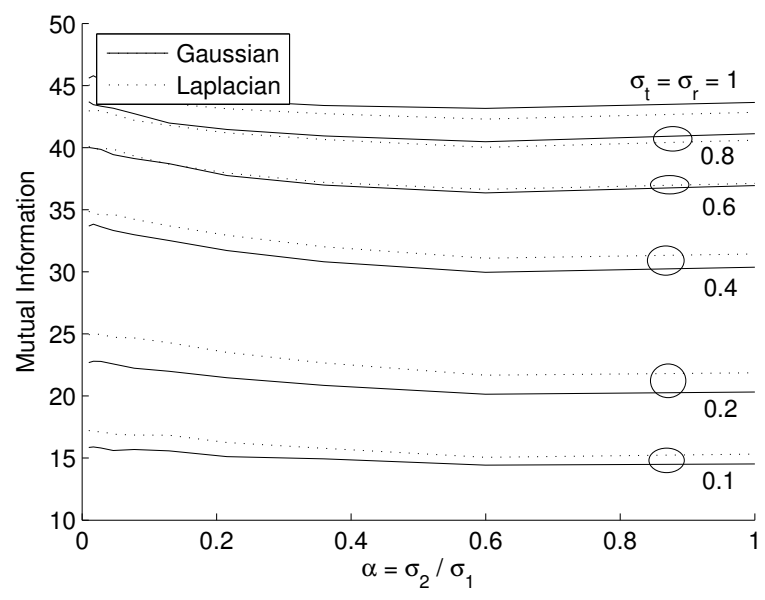

(a)

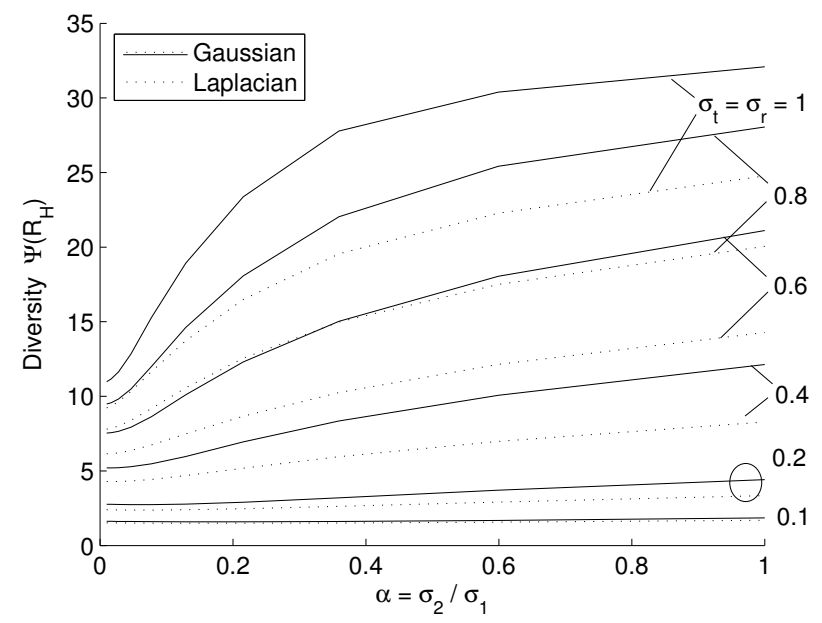

(b)

4: (a) Mutual information and (b) diversity measure for elliptical distibutions with constant $\theta_{v}=45^{\circ}$, when varying the major-minor axis ratio $\alpha$ for different transmitter/receiver angular spreads.

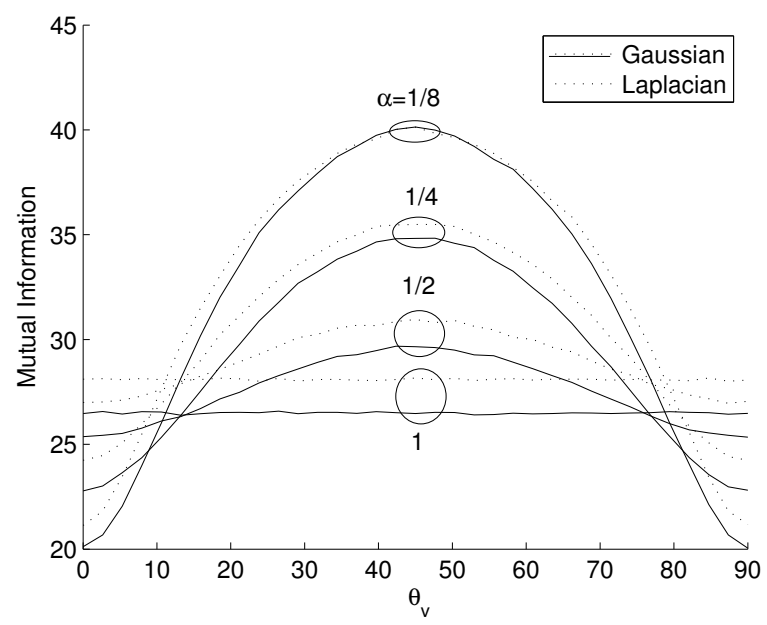

(a)

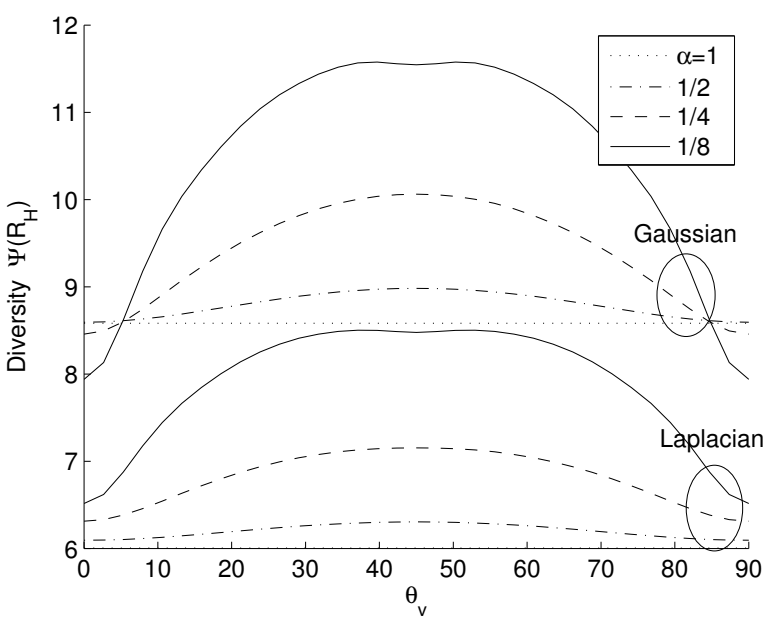

(b)

5: (a) Mutual information and (b) diversity measure for Gaussian (Laplacian) distibutions when varying the orientation angle $\theta_{v}$, for distributions with constant ellipse area $\pi \sigma_{1} \sigma_{2}=0.1 \pi(0.2 \pi)$ but differing major-minor axis ratio $\alpha$.

\section{EXAMPLES}

We now characterize some of the capacity and diversity properties of several MIMO channels generated by our MIMO model. We draw from [19] the mutual information of the MIMO channel with equally alloted transmit powers, which indicates ergodic capacity with perfect channel state information at the transmitter:

$$
I=E_{\boldsymbol{H}}\left\{\log _{2} \operatorname{det}\left(\boldsymbol{I}_{n_{T}}+\frac{\mathrm{SNR}}{n_{R}} \boldsymbol{H} \boldsymbol{H}^{\dagger}\right)\right\},
$$

where $\boldsymbol{I}_{n_{T}}$ is the $n_{T} \times n_{T}$ identity matrix and SNR the average receive signal-to-noise ratio. In following examples, SNR is set to $20 \mathrm{~dB}$ and $I$ is calculated from $\boldsymbol{R}_{\boldsymbol{H}}$ by ensemble averaging over 1000 realizations of $\boldsymbol{H}$, as generated from the method in Section IV.

Channel diversity is generally defined by the distribution of the $n_{R} n_{T}$ eigenvalues $\lambda_{i}$ of the channel correlation matrix
$\boldsymbol{R}_{\boldsymbol{H}}$. These eigenvalues describe the average powers of the independent eigenvector channels of a MIMO channel. A useful single parameter diversity measure describing the eigenvalue spread was introduced in [20]:

$$
\Psi\left(\boldsymbol{R}_{\boldsymbol{H}}\right)=\frac{\left(\sum_{i=1}^{n_{R} n_{T}} \lambda_{i}\right)^{2}}{\sum_{i=1}^{n_{R} n_{T}} \lambda_{i}^{2}}=\left(\frac{\operatorname{trace}\left\{\boldsymbol{R}_{\boldsymbol{H}}\right\}}{\left\|\boldsymbol{R}_{\boldsymbol{H}}\right\|_{F}}\right)^{2},
$$

where $\|\cdot\|_{F}$ is the Frobenius matrix norm.

With these metrics we characterize performance of a $10 \times 10$ MIMO system with antennas arranged in uniform circular arrays of radii $2 \lambda$. Fig. 4 and Fig. 5 illustrate the effect of the elliptical distribution parameters, namely the ellipse majoraxis-to-minor-axis ratio $\alpha \triangleq \sigma_{2} / \sigma_{1}$ and elliptical contour orientation $\theta_{v}$ on the channel capacity and diversity.

In Fig. 4 we study the effect of varying the ellipse majoraxis-to-minor-axis ratio, while keeping the scatterer densities around the transmitter and receiver constant, for $\theta_{v}=45^{\circ}$. The 
elliptic distribution offers a small 1 to $3 \mathrm{~dB}$ boost to capacity, while the diversity increases with $\alpha$, and is maximum for circularly-contoured angular distributions $(\alpha=1)$. Distributions with narrow elliptic contours (i.e. low $\alpha$ ) correspond to smaller diversities.

Fig. 5 studies the effect of rotating the ellipse (varying $\theta_{v}$ ) while holding the area of the contours of the power distribution constant, for different $\alpha$. Diversity and mutual information are typically maximised at $\theta_{v}=45^{\circ}$ where the angular spreads at the transmitter and receiver are equally distributed. However $\alpha=1$ (circular contours) corresponds to a low performance which is unaffected by rotation.

\section{CONCLUSION}

In this paper, we present a MIMO model for WSSUS channels, and propose bivariate Gaussian, bivariate Laplacian and Morgenstern (generalized uniform-limited) distributions for the distribution of scatterers. We explore channel capacity and diversity as a function of bivariate distribution parameters showing that, for elliptical distributions, narrow elliptical contours yield low diversity while the orientation of elliptical contours significantly influences capacity.

\section{REFERENCES}

[1] R. W. Heath Jr. and K.R. Dandekar, "Characterizations of narrowband MIMO channels," in IEEE International Symposium on Wireless Communications, Sept. 2002, Invited Paper.

[2] K. Yu, M. Bengtsson, B. Ottersten, D.P. McNamara, P. Karlsson, and M.A. Beach, "Second order statistics of NLOS indoor MIMO channels based on $5.2 \mathrm{GHz}$ measurements," in IEEE Global Communications Conf., Nov. 2001.

[3] J. P. Kermoal, L. Schumacher, K. I. Pedersen, P. E. Mogensen, and F. Frederiksen, "A stochastic MIMO radio channel model with experimental validation," IEEE J. Select. Areas Commun., vol. 20, no. 6, pp. 1211-1226, 2002

[4] T. S. Pollock, "Correlation modelliing in MIMO systems: when can we Kronecker?," in Australian Communications Theory Workshop, Newcastle, 2004, pp. 149-153.

[5] H. Ozcelik, N. Czink, and E. Bonek, "What makes a good MIMO channel model," in Vehicular Technology Conference, 2005, vol. I, pp. $156-160$
[6] W. Weichselberger, H. Ozcelik, M. Herdin, and E. Bonek, "A novel stochastic MIMO channel model and its physical interpretation," in 6th International Symposium on Wireless Personal Multimedia Communications (WPMC03), 2003.

[7] A. M. Sayeed, "Deconstructing multiantenna fading channels," IEEE Transactions on Signal Processing, vol. 50, no. 10, pp. 2563-2579, Oct. 2002.

[8] Q. H. Spencer, B. D. Jeffs, M. A. Jensen, and A. L. Swindlehurst, "Modeling the statistical time and angle of arrival characeristics of an indoor multipath channel," IEEE J. Select. Areas Commun., vol. 18, no. 3, pp. 347-360, 2000 .

[9] P. A. Bello, "Characterization of randomly time-variant linear channels," IEEE Trans. Comm. Sys., vol. 11, pp. 360-393, 1963.

[10] Ed. W. C. Jakes, "Microwave mobile communications," Wiley, New York, 1974.

[11] J. Saltz and J. H. Winters, "Effect of fading correlation on adaptive arrays in digital mobile radio," IEEE Trans. Veh. Technol., vol. 42, pp 1049-1057, 1994.

[12] R. G. Vaughan, "Pattern translation and rotation in uncorrelated source distributions," IEEE Trans. Antennas Propagat., vol. 46, no. 7, pp. 982990, 1998.

[13] K. L. Pederson, P. E. Morgensen, and B. H. Fleury, "Power azimuth spectrum in outdoor environments," IEEE Electron. Lett., vol. 33, no. 18, pp. 1583-1584, 1997.

[14] T.D Abhayapala, T.S. Pollock, and R.A. Kennedy, "Spatial decomposition of MIMO wireless channels," in Proc. Seventh International Symposium on Signal Processing and its Applications, ISSPA 2003, July 2003, vol. 1, pp. 309-312.

[15] A. Graham, Kronecker products and matrix calculus with applications, Ellis Horwood, Chichester, 1981

[16] N. L. Johnson and S. Kotz, “On some generalized Farlie-GumbelMorgenstein distribution," Communications in Statistics, vol. 4, pp. 415-427, 1975.

[17] P. Teal and T. A. Abhayapala, "Spatial correlation in non-isotropic scattering scenarios," in Proc. IEEE International Conference on Acoustics, Speech and Signal Processing, 2002, vol. III, pp. 2833-2866.

[18] T. J. Kozubowski and K. Podgorski, "A multivariate and asymmetric generalization of Laplace distribution," Computational Statistics, vol. 15, no. 4, pp. 531-540, 2000.

[19] G. J. Foschini and M. J. Gans, "On limits of wireless communications in fading environments when using multiple antennas," Wireless Personal Communications, vol. 6, pp. 311-335, 1998.

[20] M. T. Ivrlac and J. A. Nossek, "Quantifying diversity and correlation of Rayleigh fading MIMO channels," in IEEE International Symposium on Signal Processing and Information Technology, ISSPIT'03, 2003. 